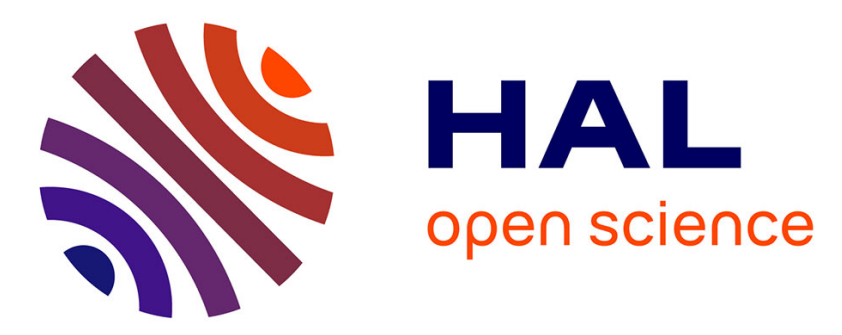

\title{
Calculation of direct correlation function for hard particles using a virial expansion
}

Michael Allen, Matthew Dennison, Andrew Masters, David Cheung

\section{To cite this version:}

Michael Allen, Matthew Dennison, Andrew Masters, David Cheung. Calculation of direct correlation function for hard particles using a virial expansion. Molecular Physics, 2009, 107 (04-06), pp.375-382. 10.1080/00268970902784934 . hal-00513261

\section{HAL Id: hal-00513261 \\ https://hal.science/hal-00513261}

Submitted on 1 Sep 2010

HAL is a multi-disciplinary open access archive for the deposit and dissemination of scientific research documents, whether they are published or not. The documents may come from teaching and research institutions in France or abroad, or from public or private research centers.
L'archive ouverte pluridisciplinaire HAL, est destinée au dépôt et à la diffusion de documents scientifiques de niveau recherche, publiés ou non, émanant des établissements d'enseignement et de recherche français ou étrangers, des laboratoires publics ou privés. 


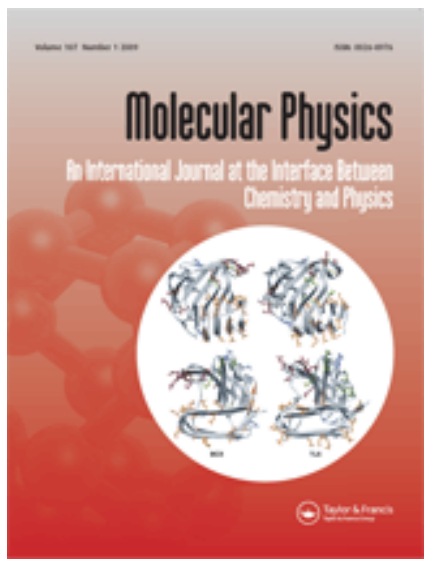

\section{Calculation of direct correlation function for hard particles using a virial expansion}

\begin{tabular}{|r|l|}
\hline Journal: & Molecular Physics \\
\hline Manuscript ID: & TMPH-2008-0447.R1 \\
\hline Manuscript Type: & Special Issue Paper - Dr. Jean-Jacques Weis \\
\hline Author: & 28-Jan-2009 \\
\hline Complete List of Authors: & $\begin{array}{l}\text { Allen, Michael; University of Warwick, Department of Physics } \\
\text { Dennison, Matthew; University of Manchester, School of Chemical } \\
\text { Engineering and Analytical Science } \\
\text { Masters, Andrew; University of Manchester, School of Chemical } \\
\text { Engineering and Analytical Science } \\
\text { Cheung, David; University of Warwick, Department of Chemistry }\end{array}$ \\
\hline Keywords: & $\begin{array}{l}\text { Monte Carlo, virial expansion, liquid structure, direct correlation } \\
\text { function }\end{array}$ \\
\hline
\end{tabular}

\section{(s) ScholaroNE" \\ Manuscript Central}


Molecular Physics

Vol. 00, No. 00, Month 200x, 1-11

\title{
RESEARCH ARTICLE
}

\section{Calculation of direct correlation function for hard particles using a virial expansion}

\author{
Matthew Dennison ${ }^{a}$, Andrew J. Masters ${ }^{a}$, David L. Cheung ${ }^{b}$ and Michael P. Allen ${ }^{c *}$ \\ ${ }^{a}$ School of Chemical Engineering and Analytical Science, University of Manchester, \\ Sackville Street, Manchester, M60 1QD, UK; \\ ${ }^{b}$ Department of Chemistry and Centre for Scientific Computing, \\ University of Warwick, Coventry, CV4 7AL, UK; \\ ${ }^{c}$ Department of Physics and Centre for Scientific Computing, \\ University of Warwick, Coventry, CV4 $7 A L, U K$ \\ (Received 00 Month 200x; final version received 00 Month 200x)
}

\begin{abstract}
We have calculated the direct correlation function, $c(1,2)$, via a high order virial expansion, for systems of hard spheres and spheroids, in both the isotropic and nematic phases. For hard spheres, we find that truncation at $6^{\text {th }}$ order in density gives good agreement with simulation data. Close to freezing, the virial series still appears to converge to the simulation results, but there are significant discrepancies, particularly at very small separations. In the non-overlap region, the virial theory begins to capture the features found from simulation. We also calculate the pair distribution function, $g(1,2)$, from our estimates of $c(1,2)$, and find good agreement with simulation data at all densities up to freezing. For hard, prolate spheroids of aspect ratio $3: 1$, we calculate $c(1,2)$ and $g(1,2)$ in the isotropic phase, again finding good agreement with simulation data at moderate densities. Finally, we present the result of our calculations on $c(1,2)$ for $3: 1$ spheroids in the nematic phase.
\end{abstract}

Keywords: Monte Carlo; virial expansion; liquid structure; direct correlation function.

\section{Introduction}

The direct correlation function, $c(1,2)$, plays a fundamental role in liquid state theory [1]. Firstly, a knowledge of this function allows one to calculate the pair distribution function via the Ornstein-Zernike relation. Secondly, it provides information as to how the Helmholtz free energy of a system changes in the presence of small spatially and orientationally dependent density fluctuations. This allows one to calculate stability limits of various phases and also leads to exact expressions for elastic constants involving integrals over $c(1,2)[2]$. Finally one can use one's knowledge of $c(1,2)$ in a uniform isotropic phase to create approximate Helmholtz free energy density functionals that can cater for inhomogeneous situations [3].

Despite this central role, in most cases it is not easy to calculate $c(1,2)$ either by simulation or theory, and so little is known about its behaviour. To our knowledge, no successful method has yet been proposed for directly measuring $c(1,2)$ from a simulation. Instead the two-particle distribution function is obtained, and then $c(1,2)$ is obtained by inverting the Ornstein-Zernike equation. Hard spheres [4] and hard spheroids (including the infinitely thin plate limit) [5-11] are among the systems studied in this way.

\footnotetext{
*Corresponding author. Email: m.p.allen@warwick.ac.uk
} 
Theoretically the most common way to calculate $c(1,2)$ is via integral equation theory [1]. Apart from the Percus-Yevick (PY) result for hard spheres, which is known analytically $[12,13]$, these equations must be solved numerically, at least for the classes of hard particles upon which we will focus in this article. When solutions to these integral equations exist, the agreement with simulation is often quite respectable, but an unfortunate problem is that all too often these equations fail to have a real solution in the most interesting regions of the phase diagram [14]. Thus the hypernetted chain (HNC) equation [15, 16], applied to the isotropic phase of hard spheroids, cannot predict the density at which the isotropic phase becomes unstable with respect to nematic fluctuations, as the solution is lost at a substantially lower density. Almost always, one cannot get close to phase transitions using these methods, and these are often the very thing in which one has the most interest.

A fundamental approach for calculating fluid properties, however, is that of using a virial expansion. This has most commonly been used to calculate the equations of state of isotropic fluids (for a recent review, see [17]). The pressure is expressed as a power series in the density and the virial coefficients are given in terms of multi-dimensional integrals involving the potential of interaction. For various hard bodies this expansion has been carried out to $10^{\text {th }}$ order for spheres [18], and $9^{\text {th }}$ order for other shapes $[19,20]$. An unsolved theoretical question is for what range of densities this expansion converges, but for hard spheres, at least, numerical studies indicate that the series converges well up to the freezing transition density and arguably is still convergent for higher densities. For example, at a packing fraction of 0.49 , very close to freezing, the tenth virial prediction of the pressure is in error by $2 \%$, whereas the eighth virial prediction is in error by $6 \%$. For aspherical hard particles the radius of convergence would appear to be considerably less than that of spheres, but the indications are that for systems which undergo an isotropicnematic liquid crystal transition, the series converges up to this transition density [21].

One may also apply the virial expansion to nematic and cubatic liquid crystalline phases $[22,23]$. This permits both a prediction of the isotropic - liquid-crystal transition density and the prediction of liquid crystalline properties, such as its equation of state and the value of the liquid crystalline order parameters. The indications are that nematic phase properties converge over a wide density range.

It is also possible, however, to calculate other properties using a virial expansion. An example is the prediction of Frank elastic constants in the nematic phase, which, formally, are given as integrals over the nematic direct correlation function [24].

Despite this progress, these virial calculations give no structural information. One way of obtaining this is to use the virial expansion to calculate the bridge function and this yields $c(1,2)$ as a result of solving integral equations. This has been carried out to 8th order in the density for hard spheres [25], and to second order in the density for spheroids [8-11, 26]. For hard spheroids, at least, this approach still suffers from the problem that the integral equations do not have real solutions in the vicinity of the phase transition. In this article we present results obtained by directly using the virial series to calculate $c(1,2)$. We present results for hard spheres and for hard spheroids in both isotropic and nematic phases and consider how well the predictions converge.

\section{Theory}

We consider a classical system of rigid particles. The combined position and orientation of particle $i$ is denoted by $i$. In general the system may be inhomogeneous, 
either as a result of an external field or because we are considering a structured phase, so the one-particle density, $\rho(i)$, may be positionally and orientationally dependent. The Ornstein-Zernike relation links $c(1,2)$ to the total correlation function, $h(1,2)$, via

$$
h(1,2)=c(1,2)+\int c(2,3) \rho(3) h(3,2) \mathrm{d} 3
$$

and the pair distribution function, $g(1,2)$, is given by

$$
g(1,2)=h(1,2)+1 \text {. }
$$

In the isotropic phase, the one-particle density is just the overall number density, $\rho=N / V$, where $N$ is the number of particles and $V$ is the volume. For spherical particles, particle orientations play no role and the direct correlation function becomes simply a function of particle separation $r_{12}=\left|\boldsymbol{r}_{1}-\boldsymbol{r}_{2}\right|$, i.e. $c(1,2) \rightarrow c\left(r_{12}\right)$. In this case one may readily obtain $h\left(r_{12}\right)$ from $c\left(r_{12}\right)$ using standard Fourier transform techniques.

For aspherical particles in an isotropic phase, the procedure is more complex. Due to the orientational dependence of $c(1,2)$, we must expand the pair functions $(c(1,2)$ and $h(1,2))$ using a spherical harmonic expansion. For axially symmetric particles, this expansion for a general function $f(1,2)$ is

$$
f(1,2)=4 \pi \sum_{\ell_{1} \ell_{2} m} f_{\ell_{1} \ell_{2} m}\left(r_{12}\right) Y_{\ell_{1}}^{m}\left(\boldsymbol{u}_{1}\right) Y_{\ell_{2}}^{-m}\left(\boldsymbol{u}_{2}\right)
$$

where $\boldsymbol{u}_{i}$ is the unit vector along the axis of particle $i$ and $Y_{\ell}^{m}\left(\boldsymbol{u}_{i}\right)$ is a spherical harmonic function. In this case, polar angles in the spherical harmonic functions are measured relative to the separation vector $\boldsymbol{r}_{12}$. To find $h(1,2)$, we must now use Hankel transforms [27] of the expansion coefficients $c_{\ell_{1} \ell_{2} m}\left(r_{12}\right)$, which we denote $\tilde{C}_{\ell_{1} \ell_{2} m}(k)$. The Ornstein-Zernike relation then takes the form

$$
\tilde{H}_{\ell_{1} \ell_{2} m}(k)=\tilde{C}_{\ell_{1} \ell_{2} m}(k)+\rho \sum_{\ell_{3}} \tilde{C}_{\ell_{1} \ell_{3} m}(k) \tilde{H}_{\ell_{3} \ell_{2} m}(k)
$$

and taking the inverse Hankel transforms of $\tilde{H}_{\ell_{1} \ell_{2} m}(k)$ gives $h_{\ell_{1} \ell_{2} m}\left(r_{12}\right)$.

Finally, in a nematic liquid crystal phase, the one-particle density takes the form

$$
\rho(1)=\rho f(1)
$$

where $f(1)$ is the one-particle orientational distribution function. For axially symmetric particles, this depends solely on the angle made between the symmetry axis of the particle and the direction of average alignment, or director, $\boldsymbol{n}$. In this case $c(1,2)$ depends on $\boldsymbol{n}$ as well as the orientations of particles 1 and 2 and their separation. The spherical harmonic expansion of a function $f(1,2)$ then takes the more general form

$$
f(1,2)=4 \pi \sum_{\ell_{1} \ell_{2} \ell m_{1} m_{2} m} f_{\ell_{1} \ell_{2} \ell m_{1} m_{2} m}\left(r_{12}\right) Y_{\ell_{1}}^{m_{1}}\left(\boldsymbol{u}_{1}\right) Y_{\ell_{2}}^{m_{2}}\left(\boldsymbol{u}_{2}\right) Y_{\ell}^{m}\left(\boldsymbol{u}_{12}\right)
$$

where $\boldsymbol{u}_{12}=\boldsymbol{r}_{12} / r_{12}$, and all angles are measured relative to an axis system based on $\boldsymbol{n}$. The procedure for inverting the Ornstein-Zernike equation to get the compo- 
nents of $h(1,2)$ is similar to, but more complicated than, that given for the isotropic phase [28].

To calculate $c(1,2)$, we consider the density expansion

$$
c(1,2)=\sum_{n=2}^{\infty} n B_{n}(1,2) \rho^{n-2} .
$$

The coefficient, $B_{n}(1,2)$ is related to the standard virial coefficient, $B_{n}$, by

$$
B_{n}=\frac{1}{V \Omega^{2}} \int B_{n}(1,2) \mathrm{d} 1 \mathrm{~d} 2
$$

where $\Omega=4 \pi$ for linear particles, and $8 \pi^{2}$ for non-linear ones. We calculate these expansion coefficients using the standard Ree-Hoover Monte Carlo methodology [29-31]. In the isotropic phase, we generate configurations of particles with random orientations. In the nematic phase we choose the orientational distributions from Onsager's trial function [32]

$$
f(1)=f(\cos (\theta))=(\alpha / 4 \pi \sinh (\alpha)) \cosh (\alpha \cos \theta) .
$$

$\alpha$ is a parameter that gives the nematic ordering of the system along the director $\boldsymbol{n}$. It ranges from 0 for isotropic ordering to $\infty$ for 'perfect' nematic ordering. $\theta$ is the angle that each particle makes with this director.

The value used for $\alpha$ should, for a self-consistent calculation, be that value which minimises the virial expansion for the Helmholtz free energy of the nematic phase [17]. If one wishes to compare results with simulation, however, another choice would be to choose a value of $\alpha$ that reproduces the observed simulation nematic order parameter.

In a standard virial calculation, one checks whether a current configuration of particles corresponds to one of the graphs that contribute to the overall virial. If it does, one accumulates the correct Ree-Hoover weighting for that graph. Repeating this step a large number of times and then averaging gives an estimate of the overall virial coefficient (either in the isotropic or nematic phase). Here we wish to calculate the expansion coefficients of $B_{n}(1,2)$ in a spherical harmonic expansion. To do this, we use histograms, each one corresponding to a term in the angular expansion. For a configuration of particles that contributes to a Ree-Hoover graph, we loop over all possible pairs of particles. For each pair we accumulate data in the histogram bin corresponding to the pair separation. The data accumulated are the products of the Ree-Hoover weighting factor with the particular combination of spherical harmonic functions required. Again after many iterations, the histogram data can be normalized to give the required expansion coefficients.

It should be noted that in the isotropic phase, this procedure duly yields the required expansion coefficients of $c(1,2)$. In the nematic phase, however, the fact that all particles are placed with orientations taken from the Onsager trial function means that one instead end up with a spherical harmonic expansion of $f(1) c(1,2) f(2)$.

A final consideration is the statistical errors in our calculations, which are inversely proportional to the square root of the number of iterations. At low-level virial theory, these errors can be negligible, but at high order, the errors become much more pronounced. This can lead to noise in our expansion at high densities, when these high-order virials become more important.

In our calculations, we use a bin width of $\mathrm{d} r_{12}=0.05$, calculating the expansion 


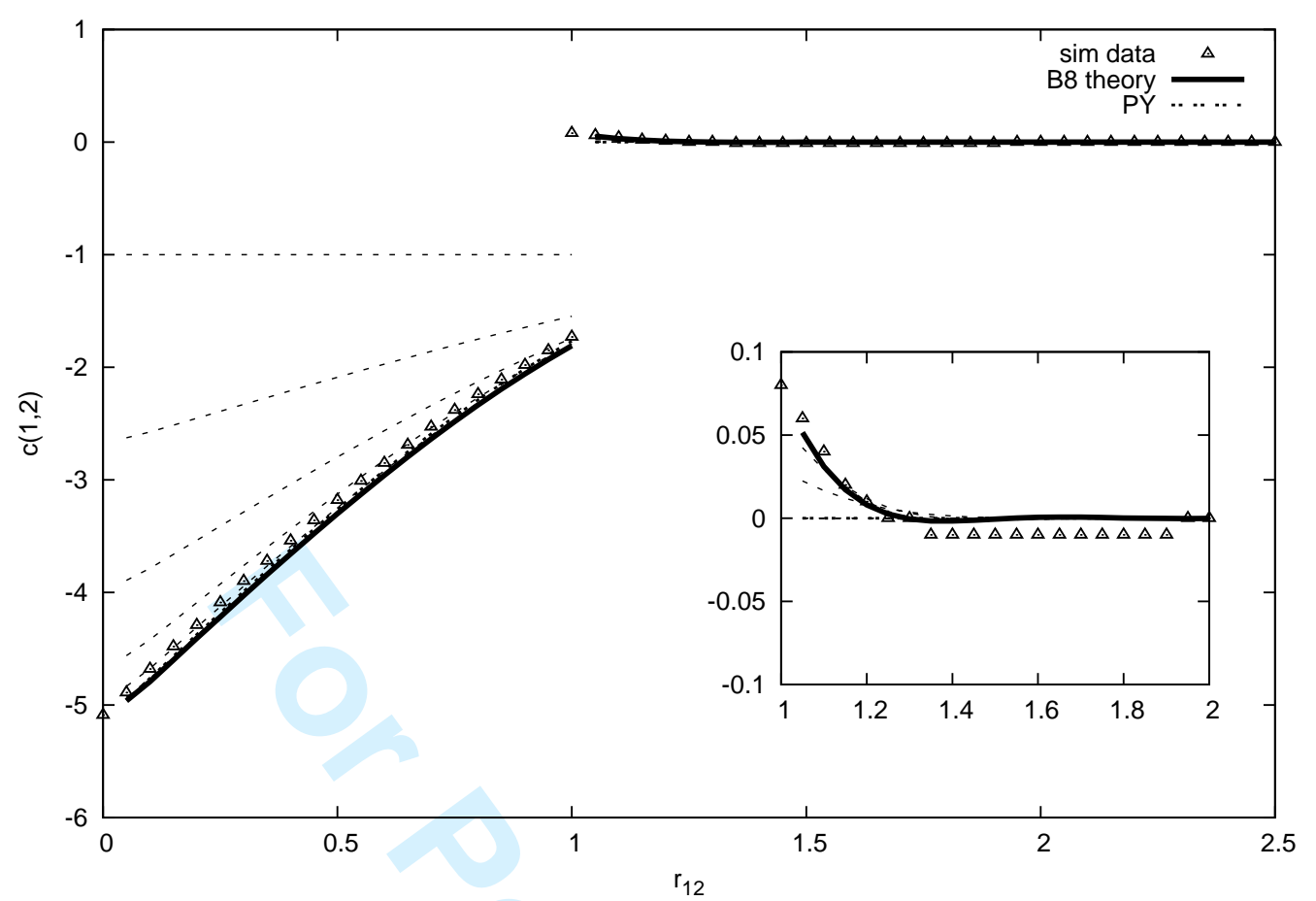

Figure 1. $c(1,2)$ for hard spheres, at $\eta=0.209$. Dotted lines are results from virial theory truncated at levels $B_{2}$ to $B_{7}$. Simulation results from [4].

coefficients up to $\ell_{\max }=6$. Typically, $10^{7}$ configurations are used for the $8^{\text {th }}$ order calculations, while at $2^{\text {nd }}$ order, we can use up to $10^{10}$ configurations.

\section{Results}

Using the above method, the contributions to $c(1,2)$ for hard spheres of diameter $\sigma$ were calculated from $2^{\text {nd }}$ to $8^{\text {th }}$ virial level. These were then used to calculate $c(1,2), h(1,2)$ and $g(1,2)$, truncated at each virial level, for a range of packing fractions. These were then compared to data obtained from simulation, as well as to the Percus-Yevick (PY) approximation. These results are shown in figures 1 to 3 .

Figure 1 shows the plot of $c(1,2)$ at $\eta=0.209$, where packing fraction $\eta=\rho v_{0}$ $\left(v_{0}\right.$ is particle volume). This is a moderate packing fraction at which an $8^{\text {th }}$-order virial expansion of the equation of state gives good agreement with simulation results (about $0.02 \%$ difference). Truncation at $2^{\text {nd }}$-order virial theory $\left(B_{2}\right)$ gives a poor estimate of $c(1,2)$, compared to simulation results, and it takes the higherorder virials to give good agreement, with the virial series converging rapidly to the simulation values. PY also gives good agreement at this packing fraction. At a higher packing fraction of $\eta=0.367$, shown in Figure 2, we still obtain very good agreement with simulation results, although the virial series converges much less rapidly, and it is only at $B_{8}$ level that the theory approaches the simulation values. However, the $B_{8}$-level theory does give better agreement than the PY approximation. At a packing fraction close to freezing, $\eta=0.4712$, shown in figure 3 , the virial series has not yet converged on the simulation results, and significantly underestimates the magnitude of $c(1,2)$, especially at small separations. This level of discrepancy is not unexpected, as at a packing fraction close to freezing, $B_{8}$ level theory underestimates the pressure by $6 \%$ and higher-order virial coefficients are needed. The PY approximation gives better agreement with simulation at this 
packing fraction for the range of $r_{12}=0$ to $r_{12}=\sigma$.

It is interesting, however, to look at $c(1,2)$ for $r_{12}>\sigma$. This behaviour is shown in the insets of figures 1 to 3 . The PY approximation gives $c(1,2)=0$ in this range. Simulation results, however, show that this is not true [4,33]. $c(1,2)$ has a positive value as one approaches $r_{12}=\sigma$ from above. At high packing fraction, one finds that this function oscillates. The virial expansion gives zero values for $c(1,2)$ at the $B_{2}$ and $B_{3}$ levels, but the higher-order terms give non-zero contributions. Adding more terms increasingly improves agreement between theory and simulation, though at the higher packing fractions, the differences are still significant and the predicted oscillations are out of phase with the simulation results. We would anticipate that the addition of a few more higher-order terms would remedy this.

Figures 4 and 5 show the calculated $g(1,2)$, for both theory and simulation, at two of the same packing fractions as above. At $\eta=0.209$ (figure 4), the agreement between simulation and theory is again excellent. The virial series converges rapidly to the simulation results, with only $B_{2}$-level theory giving particularly poor agreement. The agreement with simulation is also good at the higher packing fraction of $\eta=0.471$ (figure 5), with the convergence of the virial series being less rapid, but still approaching the simulation results for high-order virial theory. Figure 5 shows that the predicted values are slightly out of phase with the simulation values, but the theory does predict the general behaviour particularly well. The value at contact is underestimated, consistent with the underestimation of the equation of state at this order theory.

An $n^{\text {th }}$-order density expansion of $c(1,2)$ yields a prediction for $g(1,2)$ also correct to $n^{\text {th }}$ order. $g(1,2)$, however, also contains higher order terms in the packing fraction which are not exact. These incorrect terms may violate the core condition, i.e. the condition that $g(1,2)=0$ when two hard particles overlap. Looking at figures 4 and 5 we see that the core condition is indeed violated, but, as expected, the situation improves, the more coefficients are used. What is pleasing is that 


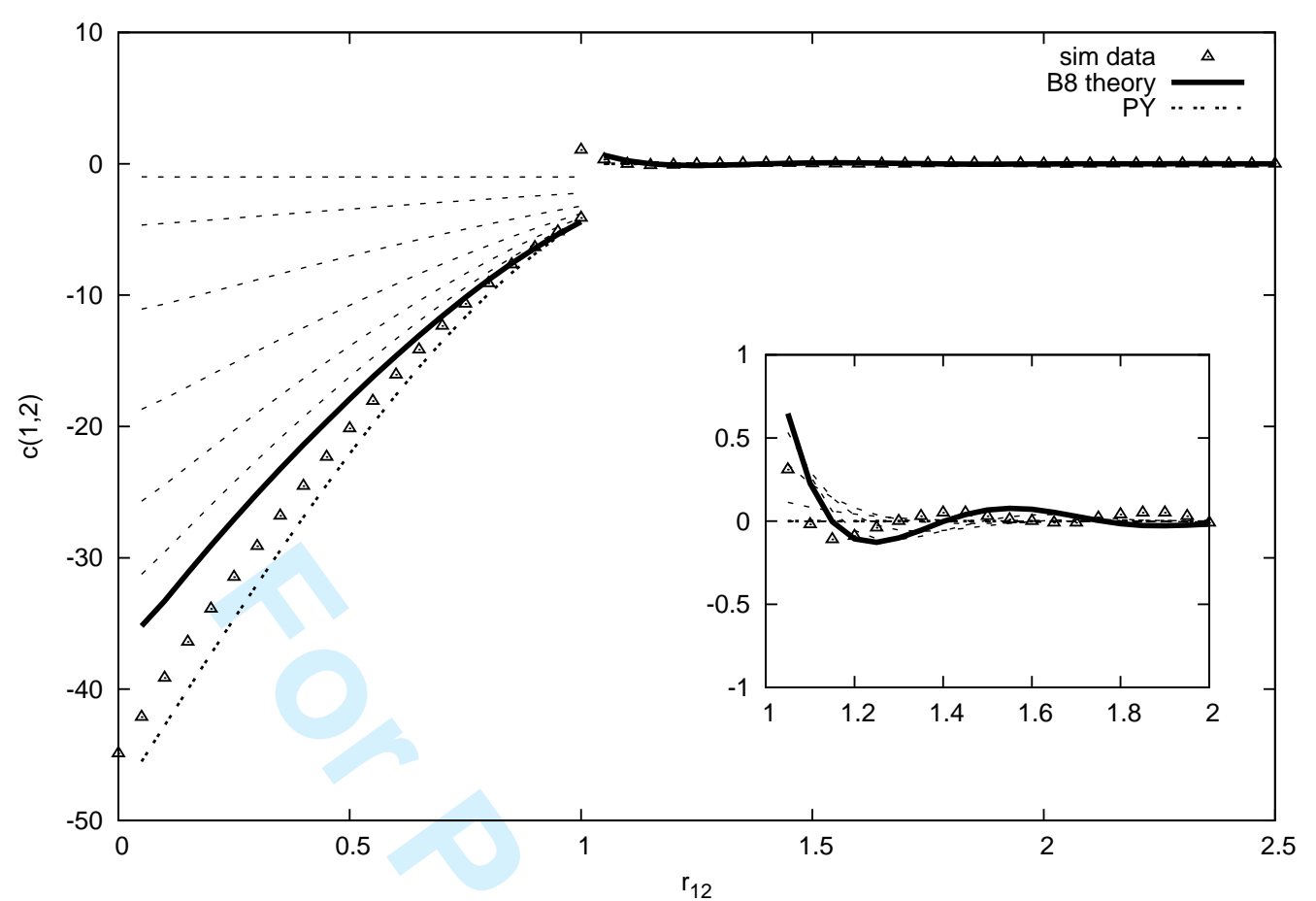

Figure 3. $c(1,2)$ for hard spheres, at $\eta=0.4712$. Dotted lines are results from virial theory truncated at levels $B_{2}$ to $B_{7}$. Simulation results from [4].

outside the overlap region, the predictions are really rather good.

Also calculated were the expansion coefficients of $c(1,2)$ and $h(1,2)$ for hard, prolate spheroids of aspect ratio $a / b=3$, where $a$ and $b$ are the length of the major and minor axes respectively. As for the hard spheres, these were calculated for truncation at different levels of virial theory, and for a variety of packing fractions. For this system, the isotropic - nematic transition is at a packing fraction of $\eta=$ 0.507 [34]. Figures 6 and 7 show the comparison of the predicted values of $c_{000}\left(r_{12}\right)$ and $c_{220}\left(r_{12}\right)$ at a packing fraction of $\eta=0.148$. Here, it can be seen that the theory provides excellent predictions of these expansion coefficients, with the virial series rapidly converging towards the simulation results. Figures 8 and 9 show these same coefficients calculated at a higher packing fraction of $\eta=0.444$. At this packing fraction the predicted values still agree somewhat with simulation, giving good predictions of the behaviour of $c_{000}\left(r_{12}\right)$ and $c_{220}\left(r_{12}\right)$, although they are somewhat off at low values of $r_{12}$. However, statistical error grows more severe for high order virials and for angular coefficients of high angular momentum number. We have not attempted to smooth these data here but this is an option that we will consider in future publications.

Using these $c(1,2)$ expansion coefficients, the expansion coefficients of $h(1,2)$ were calculated. Figures 10 and 11 show the predicted values of $h_{000}\left(r_{12}\right)$ and $h_{220}\left(r_{12}\right)$ at a packing fraction of $\eta=0.148$, compared to values obtained froom simulation. At this packing fraction, the agreement between simulation and theory is again very good for both, although as for hard spheres, the core condition is again violated (the condition in this case is that $h_{000}\left(r_{12}\right)=-1$ ). The plot of $h_{220}\left(r_{12}\right)$ (Fig. 11) shows that the predicted values are somewhat off from simulation results, most likely due to the truncation of the expansion coefficients at $\ell_{\max }=6$. It would be hoped that calculating to a higher $\ell_{\max }$ would help overcome this problem. The effects of truncation at $\ell_{\max }=6$ becomes more pronounced at higher packing fractions, as can be seen in Figures 12 and 13. Here, at $\eta=0.444$, the series appears 


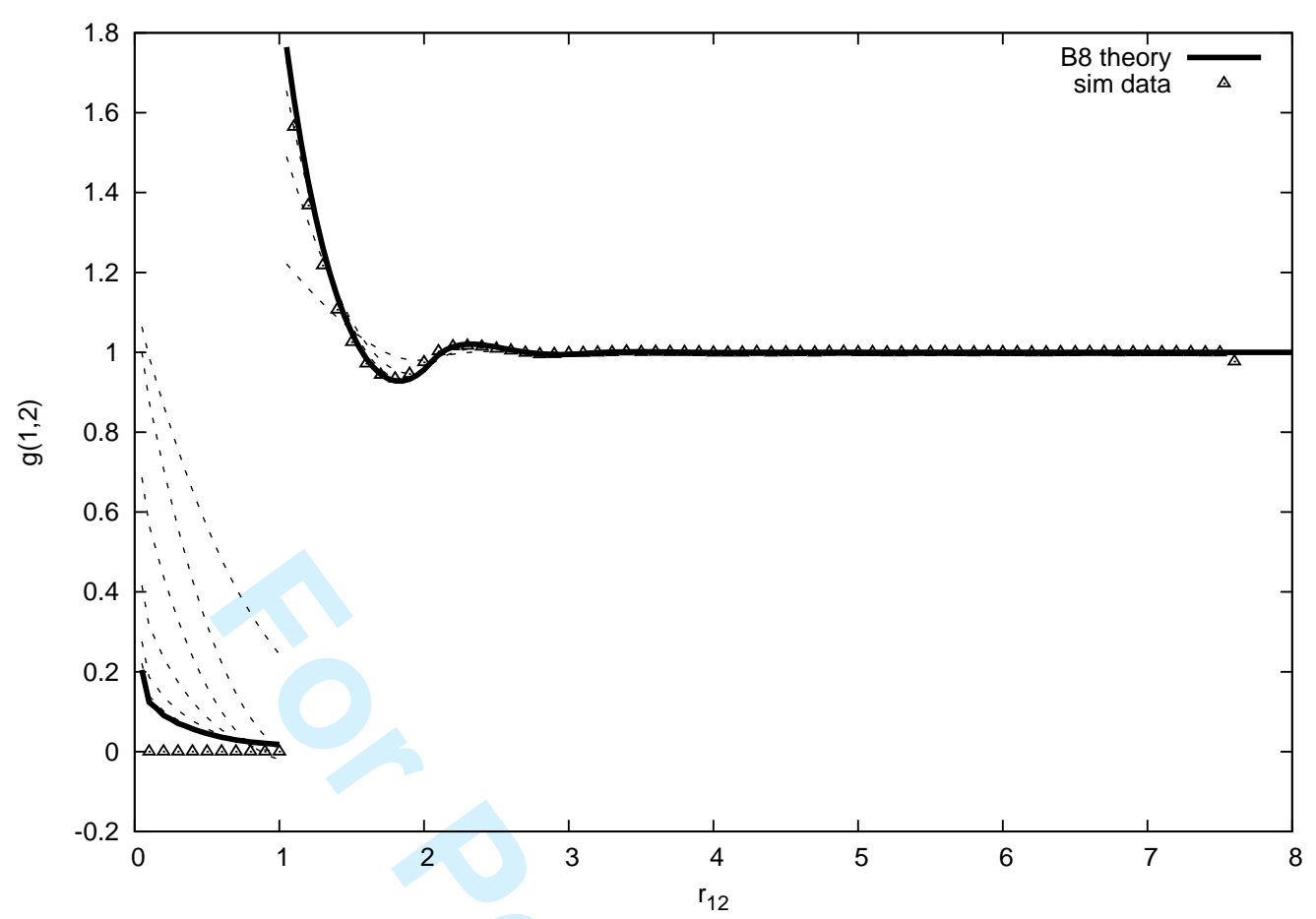

Figure 4. $g(1,2)$ for hard spheres, at $\eta=0.209$. Dotted lines are results from virial theory truncated at levels $B_{2}$ to $B_{7}$

to be converging towards the simulation data, but even at $B_{8}$ level, it is still quite a way off, although it does capture the essential behaviour of the coefficients.

Finally, the expansion coefficients of $f(1) c(1,2) f(2)$ were calculated for the same system as above. Taking the value of $\alpha=13.92$, corresponding to a nematic order parameter $S_{2}=\left\langle P_{2}(\boldsymbol{u} \cdot \boldsymbol{n})\right\rangle=0.8$ (where $P_{2}$ is the second Legendre polynomial), from a virial equation of state for a packing fraction of $\eta=0.694$, the expansion coefficients of $f(1) c(1,2) f(2)$ were calculated for this value, and also, for comparison, $\alpha=0$, which corresponds to an isotropic system. The values of $c_{000000}\left(r_{12}\right)$ are shown in figure 14 . The behavior of $c_{000000}\left(r_{12}\right)$ for the nematic system is visibly different from an isotropic system at the same density. At low $r_{12}$, the magnitude of $c_{000000}\left(r_{12}\right)$ for the nematic system is lower than that of the isotropic system. This is expected, due to the high orientational ordering of the system causing more parallel particles. This in turn lowers the chance of overlapping particles of a shortto mid-range separation. However, at larger separations, the magnitude in the nematic system becomes larger than that for the isotropic system, as the ordered nature of the system makes overlaps of particles of this separation more likely. The inversion of these data to obtain $h(1,2)$ for the nematic phase is work in progress.

\section{Conclusions}

We have calculated the virial expansion of the direct correlation function to $6^{\text {th }}$ order in the density for both hard spheres and hard prolate $3: 1$ spheroids. In the isotropic phase, our results show that the series appears to be convergent for hard spheres up to the freezing density and for the hard spheroids up to densities close to the isotropic-nematic phase transition. The $6^{\text {th }}$ order results compare well with simulation data up to moderate densities, but for hard spheres close to freezing, there are significant differences, especially at small particle separations. Given that the pressure predicted from the pressure virial series at this level of truncation 


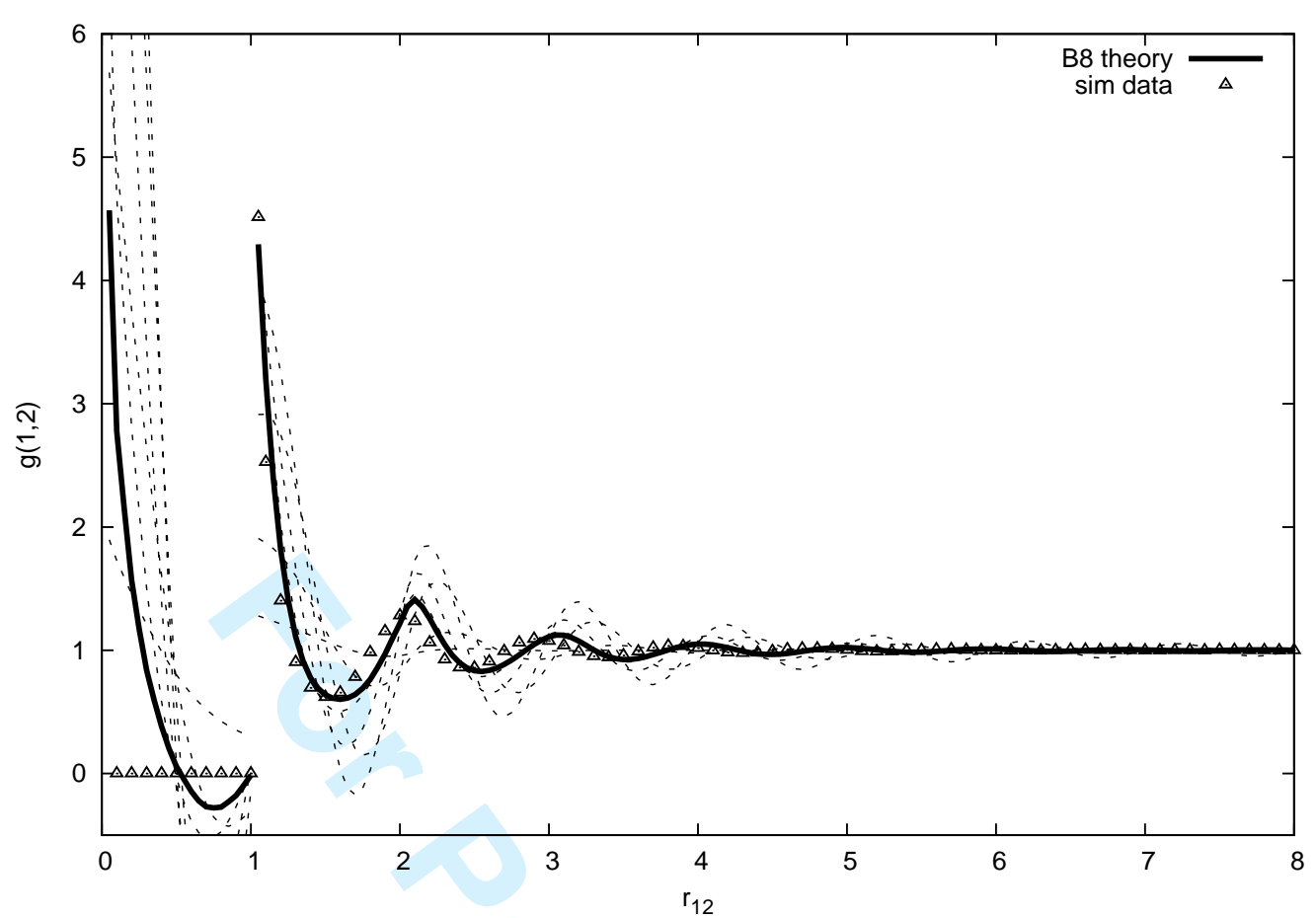

Figure 5. $g(1,2)$ for hard spheres, at $\eta=0.4712$. Dotted lines are results from virial theory truncated at levels $B_{2}$ to $B_{7}$

is underestimated by $6 \%$, such discrepancies are not surprising. Simulation shows that the direct correlation function has an oscillatory behaviour in the non-overlap region. This is reproduced by the virial expansion, though more terms are needed in the expansion in order to get good agreement with the phase of oscillation. The question of whether or not the asymptotic long-range behaviour of both $g(1,2)$ and $c(1,2)$ is oscillatory is also of great interest $[35,36]$ and merits further study.

The Ornstein-Zernike relation was used to calculate the pair distribution function, $g(1,2)$, from our direct correlation function data. Outside the overlap region, the agreement with simulation results is excellent, even for hard spheres close to freezing. Within the overlap region, however, the pair distribution function is not zero as it should be. For spheres, adding more terms in the virial expansion progressively reduces this discrepancy and at $6^{\text {th }}$ order, the core condition is fairly well obeyed. For spheroids, one must consider both the truncation of the virial series and also the truncation of the angular expansion of $c(1,2)$ in spherical harmonics. We believe that the violation of the core condition is associated with both these truncations and, in a future publication, we will present a systematic study of the effects of including higher order angular terms.

In summary, we have shown that the $6^{\text {th }}$ virial expansion is highly effective at predicting accurate values for $c(1,2)$ up to moderately high densities and there is every indication that adding higher-order terms will improve the situation still further. This function may then be used to predict high quality estimates of $g(1,2)$, outside the overlap region, even at high densities. As discussed earlier, an $n^{\text {th }}$ order expansion in the density for $c(1,2)$ generates $g(1,2)$ to all orders in the density, though only the first $n$ terms are exact. These higher-order terms cause our predictions for $g(1,2)$ to be superior to those for $c(1,2)$. Unlike integral equation theory, this method does not suffer from loss of solution near a phase transition and it thus provides theoretical tools for studying structural changes in these interesting regions.

We finally presented preliminary results for $c(1,2)$ in the nematic phase. Again 


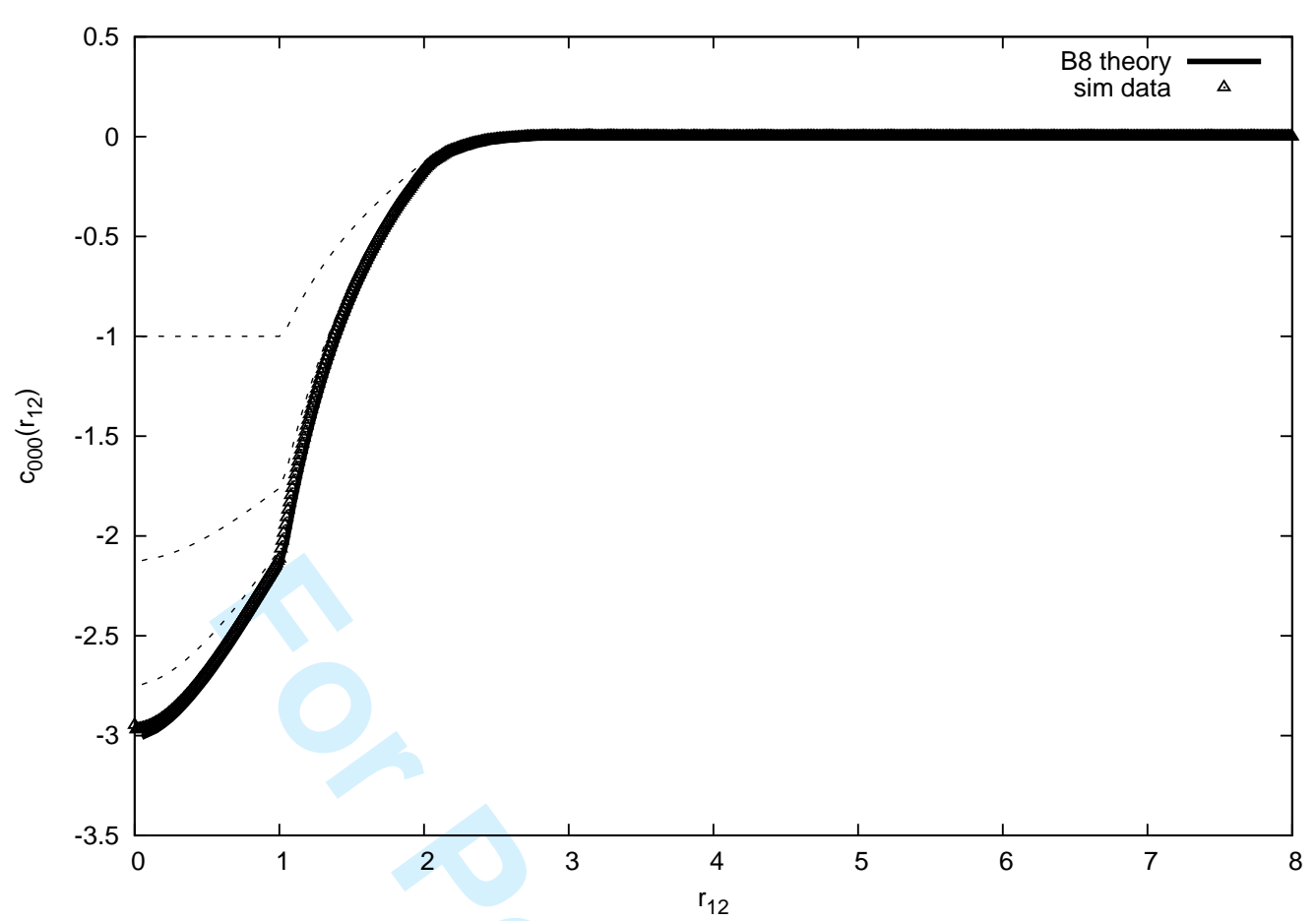

Figure 6. $c_{000}\left(r_{12}\right)$ at $\eta=0.148$ for hard, prolate spheroids of aspect ration $3: 1$. Dotted lines are results from virial theory truncated at levels $B_{2}$ to $B_{7}$

the indications are that the virial series converges well with increased number of virials. To date, integral equation theory is not well-developed for use in the nematic phase and reports vary as to whether nematic solutions exist [37-40]. The virial expansion, however, does not suffer from such difficulties and, based on our $c(1,2)$ calculations, we will present virial predictions for $g(1,2)$ in the nematic phase in a future publication.

\section{Acknowledgements}

The authors gratefully acknowledge the UK EPSRC for providing funding for MD and DC, the University of Manchester and the University of Warwick for computer time, and Marcus Bannerman for supplying simulation $g(1,2)$ functions. Computing facilities were provided by the Warwick University Centre for Scientific Computing.

\section{References}

[1]J.P. Hansen and I.R. McDonald, Theory of Simple Liquids, 3rd ed. (Academic Press, London, 2006).

[2]A. Poniewierski and J. Stecki, Molec. Phys. 38, 1931-1940 (1979).

[3] A.D.J. Haymet, Ann. Rev. Phys. Chem. 38, 89-108 (1987).

[4]R.D. Groot, J.P. van der Eerden and N.M. Faber, J. Chem. Phys. 87, 2263-2270 (1987).

[5] M.P. Allen, C.P. Mason, E. de Miguel and J. Stelzer, Phys. Rev. E 52, R25-R28 (1995).

[6]J. Stelzer, M.A. Bates, L. Longa and G.R. Luckhurst, J. Chem. Phys. 107, 7483-7492 (1997).

[7] N.H. Phuong and F. Schmid, J. Chem. Phys. 119, 1214-1222 (2003).

[8]D.L. Cheung, L. Anton, M.P. Allen and A.J. Masters, Phys. Rev. E 73, 061204/1-10 (2006).

[9]—, Phys. Rev. E 76, 041201/1-8 (2007).

[10]_ Comput. Phys. Commun. 179, 61-65 (2008).

[11]D.L. Cheung, L. Anton, M.P. Allen et al., Phys. Rev. E 78, 041201/1-13 (2008).

[12] M.S. Wertheim, Phys. Rev. Lett. 10, 321-323 (1963).

[13]E. Thiele, J. Chem. Phys. 39, 474-479 (1963).

[14]L. Belloni, J. Chem. Phys. 98, 8080-8095 (1993).

[15] A. Perera, P.G. Kusalik and G.N. Patey, J. Chem. Phys. 87, 1295-1306 (1987). 


\section{Page 11 of 15

Figure 7. $c_{220}\left(r_{12}\right)$ at $\eta=0.148$ for hard, prolate spheroids of aspect ration $3: 1$. Dotted lines are results from virial theory truncated at levels $B_{2}$ to $B_{7}$

[16] A. Perera, G.N. Patey and J.J. Weis, J. Chem. Phys. 89, 6941-6946 (1988).

[17] A.J. Masters, J. Phys. Cond. Mat. 20, 283102/1-10 (2008).

[18] N. Clisby and B.M. McCoy, J. Stat. Phys. 122, 15-57 (2006).

[19]X.M. You, A.Y. Vlasov and A.J. Masters, J. Chem. Phys. 123, 034510/1-7 (2005).

[20]P. Moravek, J. Kolafa and M. Francova, Collect. Czech. Chem. Comm. 73, 459-480 (2008).

[21]X.M. You, A.Y. Vlasov, L. Anton and A.J. Masters, Phys. Rev. E (2009), (submitted).

[22] M.A. Bates, M. Dennison and A.J. Masters, J. Chem. Phys. 129, 074901/1-10 (2008).

[23]P.D. Bates, M. Dennison, A.J. Masters and M.R. Wilson, Phys. Rev. E (2009), (submitted).

[24]P.A. O'Brien, M.P. Allen, D.L. Cheung et al., Phys. Rev. E 78, 051705/1-7 (2008).

[25] J. Kolafa and S. Labík, Molec. Phys. 104, 1915-1924 (2006).

[26]D.L. Cheung, L. Anton, M.P. Allen and A.J. Masters, Phys. Rev. E 77, 011202/1-8 (2008).

[27]L. Blum and A.J. Torruella, J. Chem. Phys. 56, 303-310 (1972).

[28] A. Perera, Phys. Rev. E 60, 2912-2929 (1999).

[29]F.H. Ree and W.G. Hoover, J. Chem. Phys. 40, 939-950 (1964).

[30]-, J. Chem. Phys. 46, 4181-4197 (1967).

[31] A.Y. Vlasov, X.M. You and A.J. Masters, Molec. Phys. 100, 3313-3324 (2002).

[32]L. Onsager, Ann. N. Y. Acad. Sci. 51, 627 (1949).

[33] D. Henderson and E.W. Grundke, J. Chem. Phys. 63, 601-607 (1975).

[34] D. Frenkel, B.M. Mulder and J.P. McTague, Phys. Rev. Lett. 52, 287-290 (1984).

[35] M. Dijkstra and R. Evans, J. Chem. Phys. 112, 1449-1456 (2000).

[36]S.V. Savenko and M. Dijkstra, Phys. Rev. E 72, 021202 (2005).

[37]H. Zhong and R.G. Petschek, Phys. Rev. E 51, 2263-2271 (1995).

[38] M.F. Holovko and T.G. Sokolovska, J. Molec. Liquids 82, 161-181 (1999).

[39] P. Mishra and Y. Singh, Phys. Rev. Lett. 97, 177801/1-4 (2006).

[40] P. Mishra, S.L. Singh, J. Ram and Y. Singh, J. Chem. Phys. 127, 044905/1-11 (2007). 


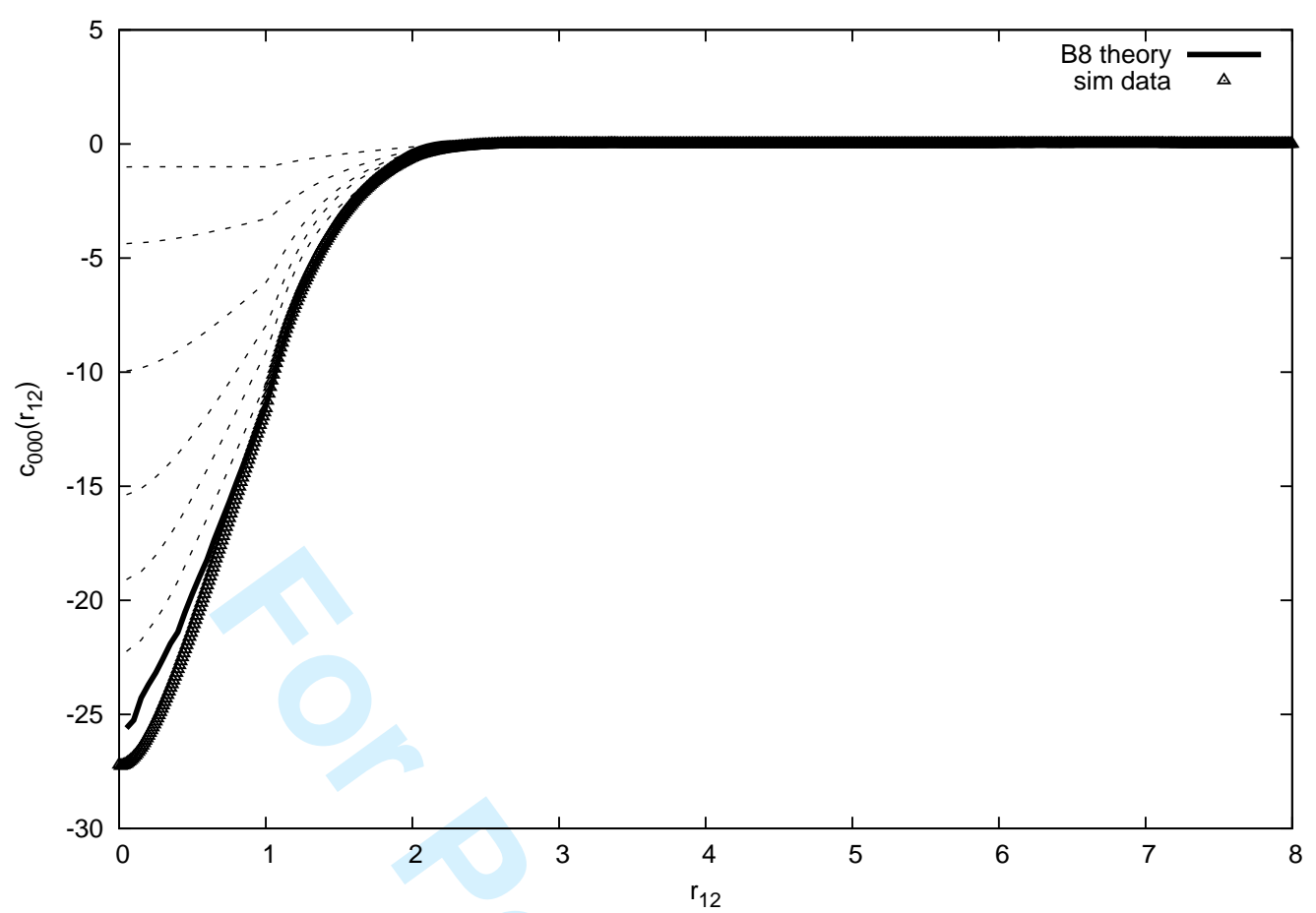

Figure 8. $c_{000}\left(r_{12}\right)$ at $\eta=0.444$ for hard, prolate spheroids of aspect ration $3: 1$. Dotted lines are results from virial theory truncated at levels $B_{2}$ to $B_{7}$

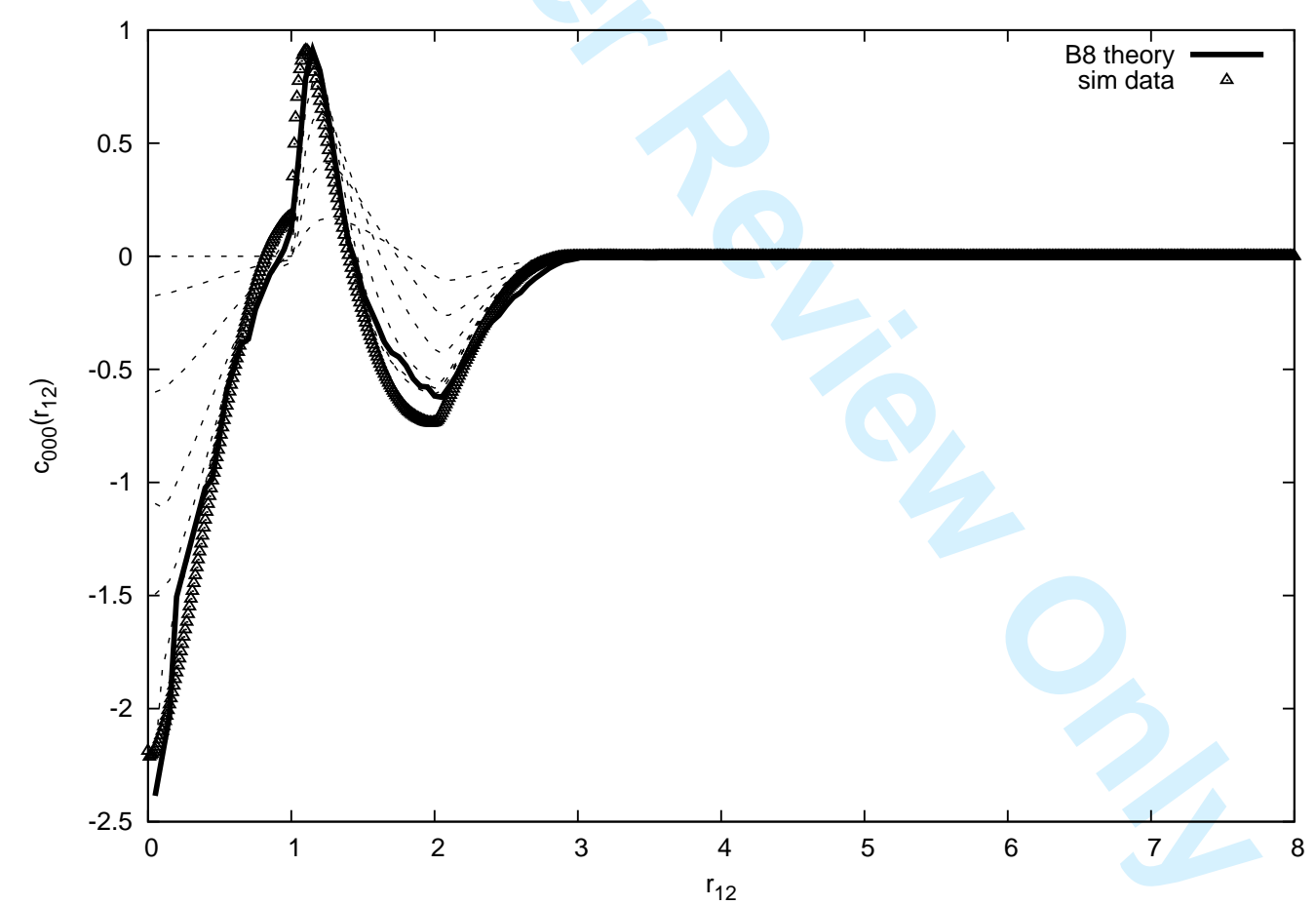

Figure 9. $\quad c_{220}\left(r_{12}\right)$ at $\eta=0.444$ for hard, prolate spheroids of aspect ration $3: 1$. Dotted lines are results from virial theory truncated at levels $B_{2}$ to $B_{7}$

2 
1

3

4

5

6

7

8

9

10

11

12

13

14

15

16

17

18

19

20

21

22

23

24

25

26

27

28

29

30

31

32

33

34

35

36

37

38

39

40

41

42

43

44

45

46

47

48

49

50

51

52

53

54

55

56

57

58

59

60

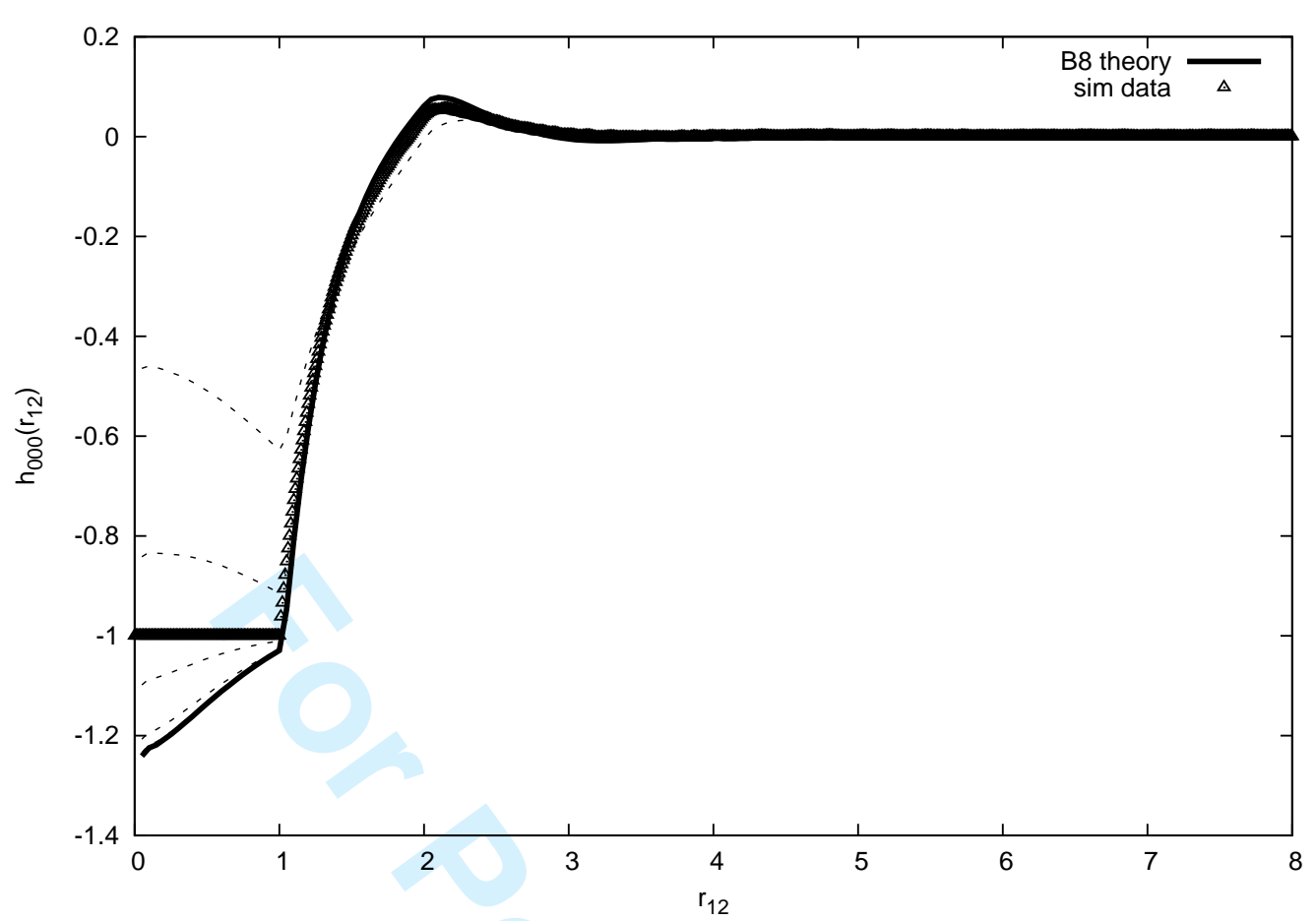

Figure 10. $h_{000}\left(r_{12}\right)$ at $\eta=0.148$ for hard, prolate spheroids of aspect ration $3: 1$. Dotted lines are results from virial theory truncated at levels $B_{2}$ to $B_{7}$

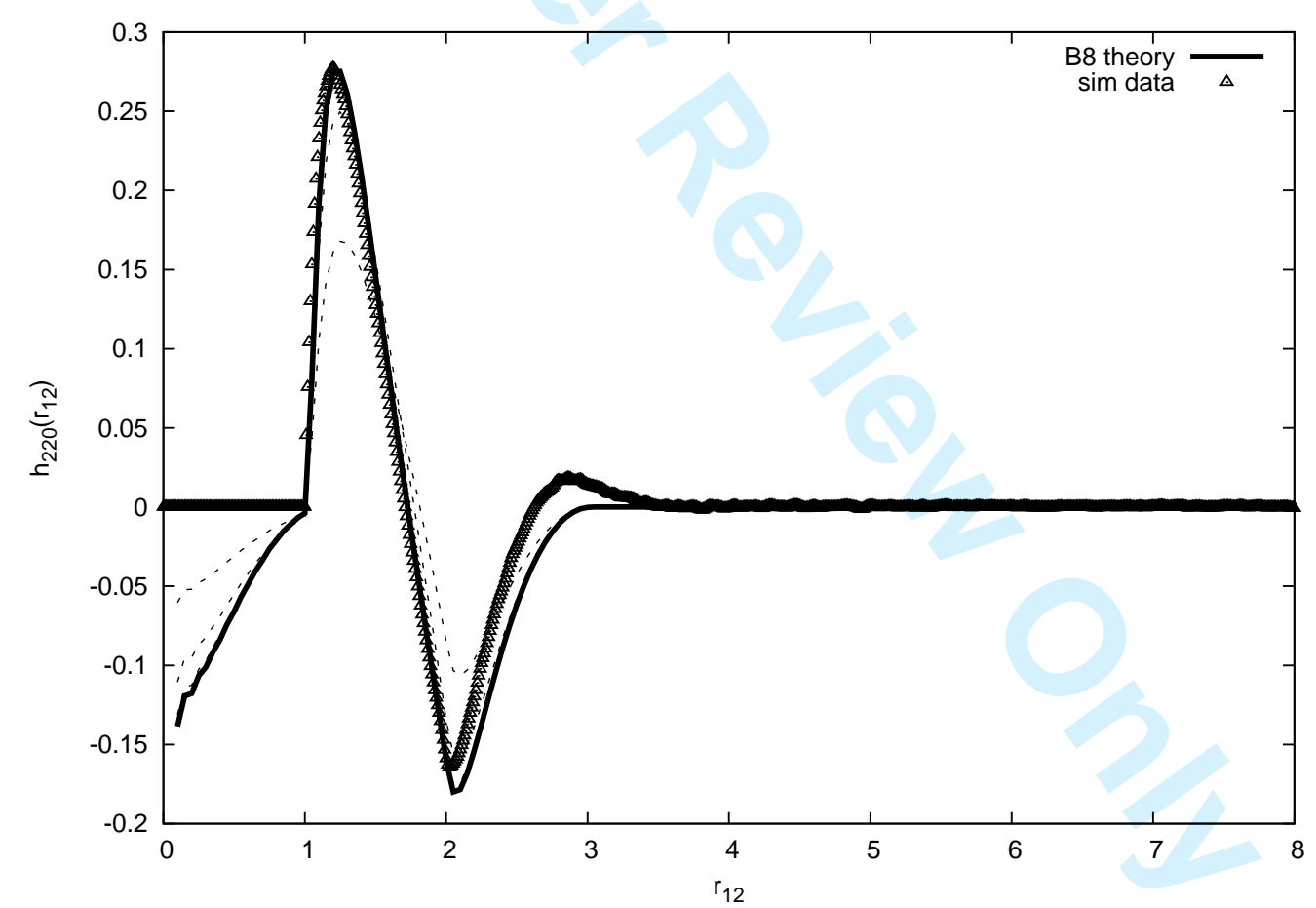

Figure 11. $h_{220}\left(r_{12}\right)$ at $\eta=0.148$ for hard, prolate spheroids of aspect ration $3: 1$. Dotted lines are results from virial theory truncated at levels $B_{2}$ to $B_{7}$ 


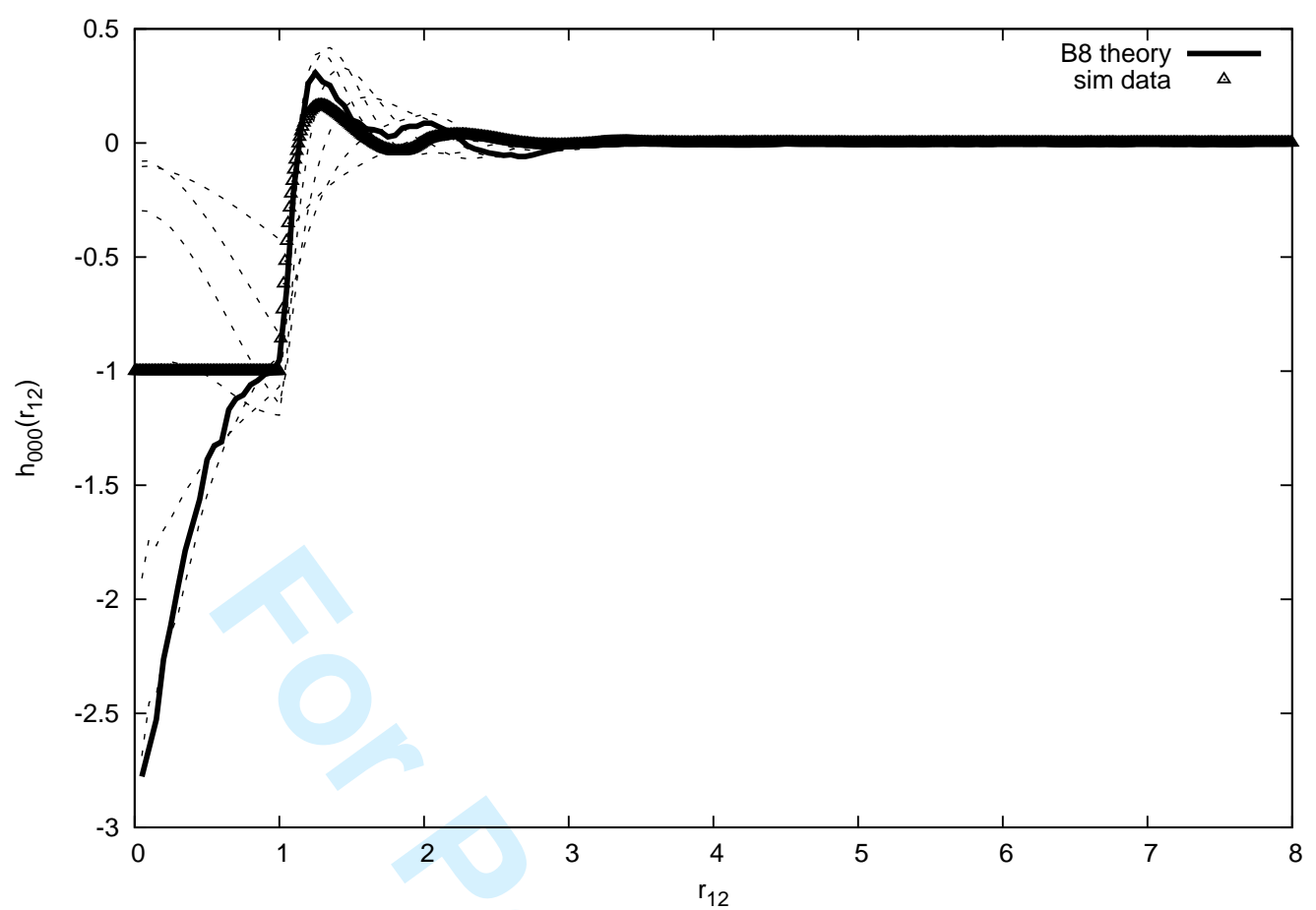

Figure 12. $h_{000}\left(r_{12}\right)$ at $\eta=0.444$ for hard, prolate spheroids of aspect ration $3: 1$. Dotted lines are results from virial theory truncated at levels $B_{2}$ to $B_{7}$

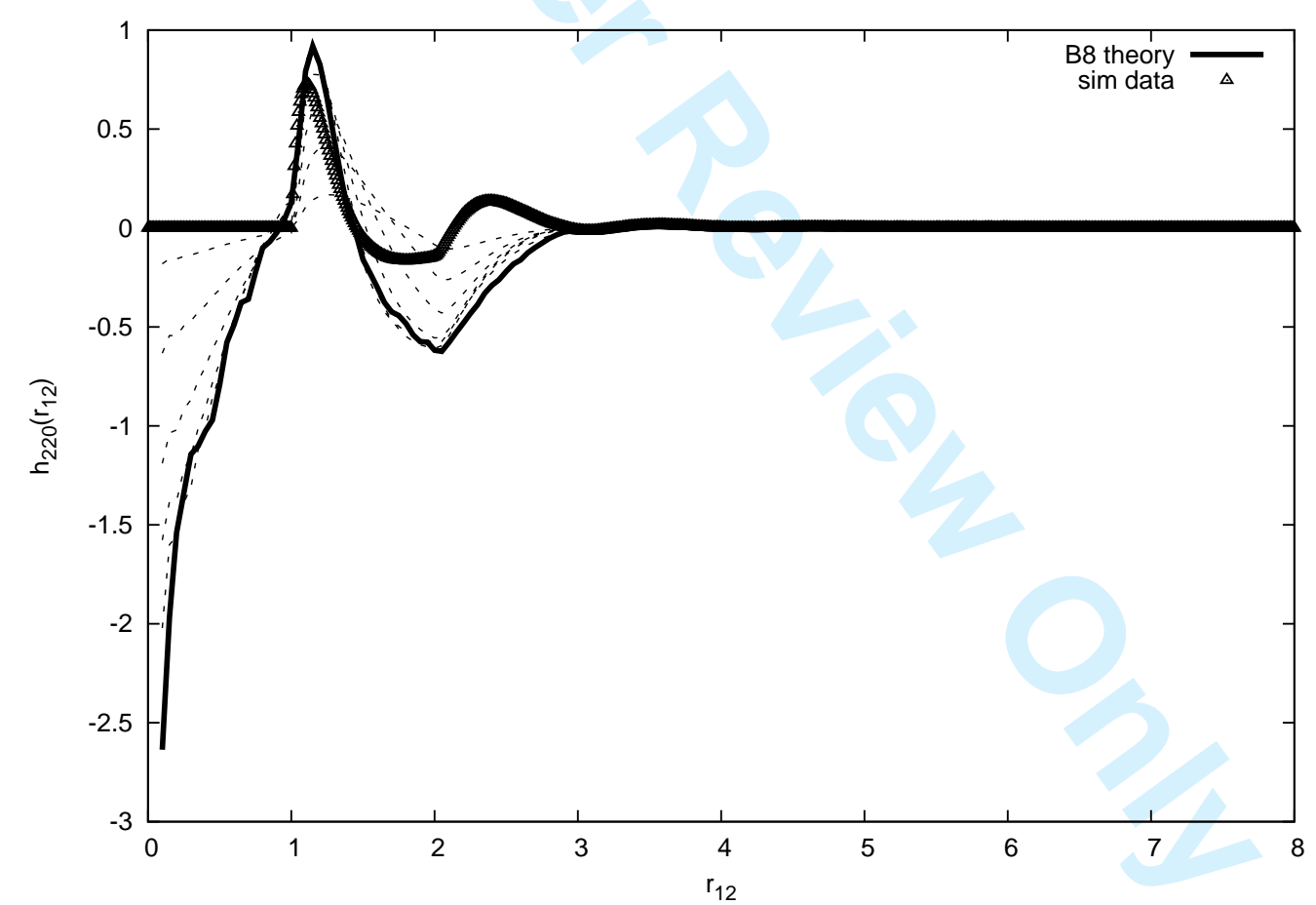

Figure 13. $h_{220}\left(r_{12}\right)$ at $\eta=0.444$ for hard, prolate spheroids of aspect ration $3: 1$. Dotted lines are results from virial theory truncated at levels $B_{2}$ to $B_{7}$

2 
1

3

4

5

6

7

8

9

10

12

13

14

15

16

17

18

19

20

21

22

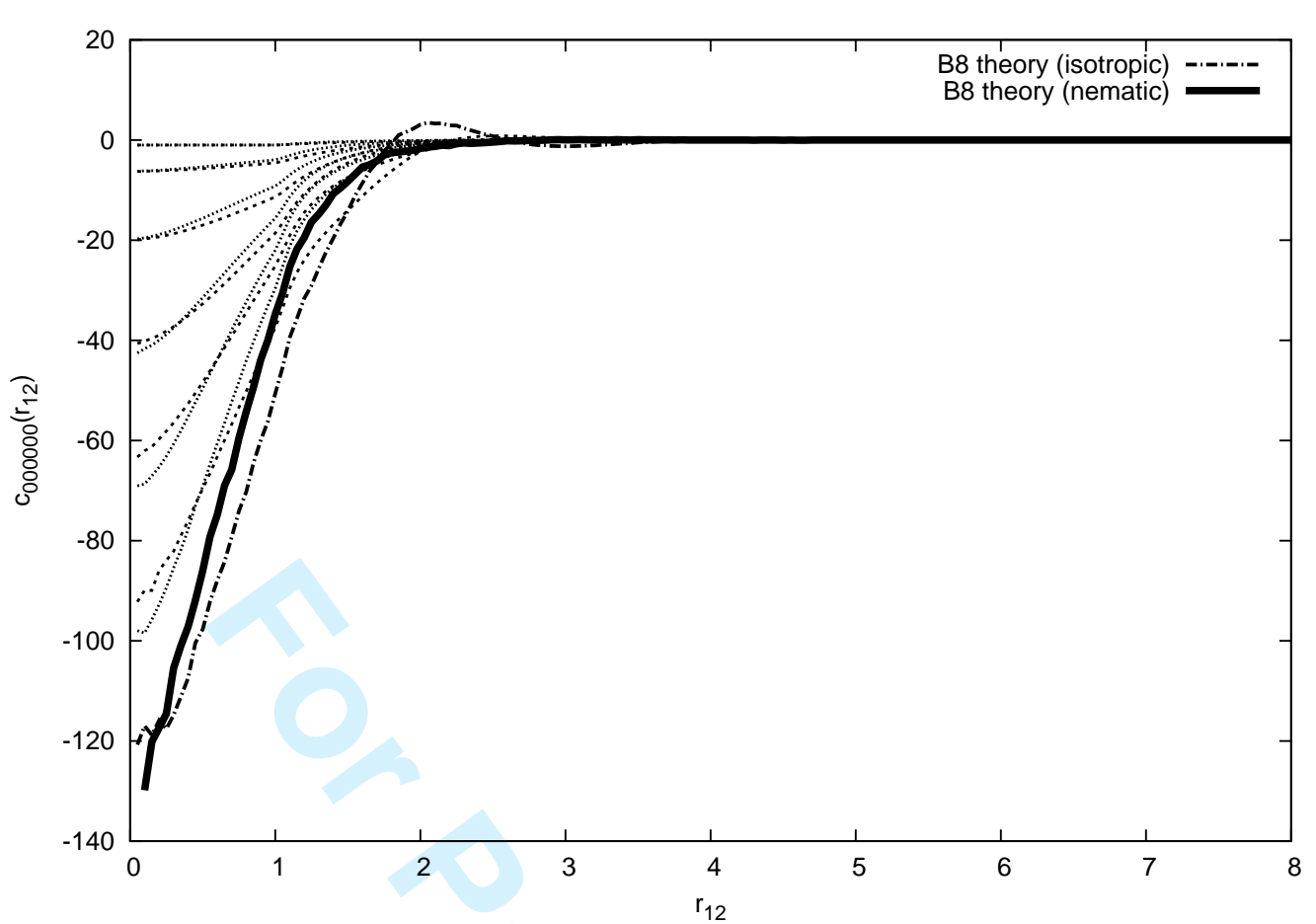

Figure 14. $c_{000000}\left(r_{12}\right)$ at $\eta=0.694$ for hard, prolate spheroids of aspect ration $3: 1$, for both isotropic and nematic ordering. Dotted lines are results from virial theory truncated at levels $B_{2}$ to $B_{7}$ for isotropic ordering, solid lines for nematic ordering. 